\title{
Full Poincaré beams obtained by means of uniaxial crystals
}

Juan Carlos G. de Sande, Massimo Santarsiero, Gemma Piquero

\section{SPIE.}




\title{
Full Poincaré beams obtained by means of uniaxial crystals
}

\author{
Juan Carlos G. de Sande ${ }^{a}$, Massimo Santarsiero $^{b}$ and Gemma Piquero ${ }^{c}$ \\ ${ }^{a}$ ETSIS de Telecomunicación, Universidad Politécnica de Madrid, Campus Sur 28031 Madrid, \\ Spain; \\ ${ }^{b}$ Dipartimento di Ingegneria, Università Roma Tre, Via V. Volterra 62, 00146 Rome, Italy; \\ ${ }^{c}$ Departamento de Óptica, Universidad Complutense de Madrid, Ciudad Universitaria, 28040 \\ Madrid, Spain
}

\begin{abstract}
The generation of non-uniformly totally polarized beams and the study of their applications is a subject of increasing interest in the last years. A particular class of beams of this kind are the so-called full Poincaré beams, which have the property of presenting all possible polarization states across their transverse section. Here we present a simple and easy way to obtain a beam endowed with such property. The method is based on the use of an initially linearly polarized beam that propagates along the optic axis of a uniaxial crystal.
\end{abstract}

\section{INTRODUCTION}

In the last decades, a great effort has been devoted to designing, generating, and analyzing light sources with special polarization characteristics and, in particular, point-dependent polarization states. ${ }^{1-25}$ Recently, a class of beams of this kind, the so-called full Poincaré beams (FPBs, for short), ${ }^{9}$ has been defined as those presenting all possible states of polarization of totally polarized light across their transverse section. More or less sophisticated methods have been proposed to generate such beams, such as those based on the use of symmetrically stressed windows ${ }^{9}$ or spatial light modulators. ${ }^{10,21,22}$

In this work, we propose a simple and easy method to obtain a FPB. We consider the field produced by a linearly polarized point-like source and propagating near the optic axis of a uniaxial crystal. Due to the different propagation features of the ordinary and extraordinary field components inside the crystal, a fully but nonuniformly polarized beam is obtained at the exit face of the crystal. ${ }^{4,26}$ Aim of this work is to show that, across its transverse section, such beam contains all the polarization states present on the Poincaré sphere and, therefore, it is a FPB.

\section{THEORETICAL BASIS}

Let us consider a uniaxial crystal of length $l$ with its optic axis along the $z$ direction of a suitable reference frame and plane parallel input and output faces perpendicular to such axis (see Fig. 1). We will assume a point-like light source at the entrance face of the crystal and consider paraxial propagation along the $z$ axis. The source is totally polarized, with linear polarization along the $x$ direction.

The field at any point $(r, \theta)$ of the exit face of the crystal can be decomposed into an extraordinary wave (the component of the electric field in the principal plane) and an ordinary wave (the component of the electric field perpendicular to the principal plane). ${ }^{26}$ Neglecting reflection losses and changes in the direction of propagation at the boundaries, these components are (omitting the temporal dependence for brevity)

$$
\begin{aligned}
& \mathbf{E}_{\mathbf{e}}(r, \theta)=E_{0}(r, \theta) \cos \theta \exp \left[\mathrm{i} k n(\alpha) d_{e}\right] \mathbf{u}_{r}, \\
& \mathbf{E}_{\mathbf{o}}(r, \theta)=-E_{0}(r, \theta) \sin \theta \exp \left(\mathrm{i} k n_{o} d_{o}\right) \mathbf{u}_{\theta},
\end{aligned}
$$

Further author information: (Send correspondence to J.C.G.S.)

J.C.G.S.: E-mail: jcgsande@ics.upm.es

G.P.: E-mail: piquero@ucm.es

M.S.: E-mail: massimo.santarsiero@uniroma3.it

Third International Conference on Applications of Optics and Photonics, edited by Manuel F. M. Costa,

Proc. of SPIE Vol. 10453, 1045323 - @ 2017 SPIE · CCC code: 0277-786X/17/\$18 · doi: 10.1117/12.2275812

Proc. of SPIE Vol. 10453 1045323-1 


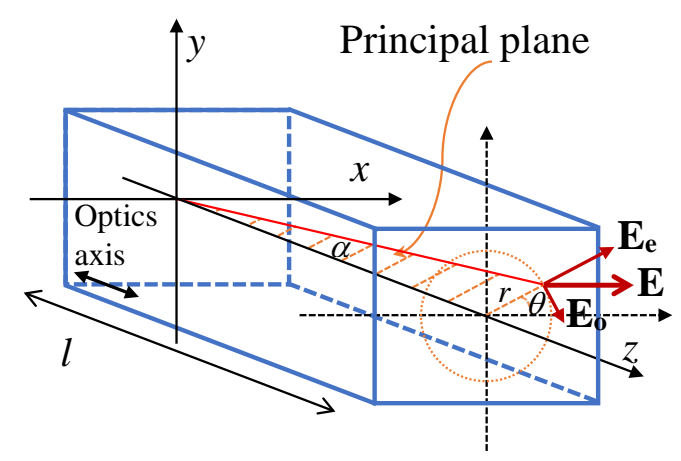

Figure 1. Crystal geometry and reference system.

where $E_{0}(r, \theta)$ is the real positive amplitude of the field at the point $(r, \theta)$ of the exit plane, $\mathbf{u}_{r}$ and $\mathbf{u}_{\theta}$ are orthonormal vectors along radial and azimuthal directions, respectively (see Fig. 1), $k$ is the wavenumber in vacuum, $n_{o}$ the ordinary refractive index and $n(\alpha)$ the refractive index for an extraordinary wave, whose propagation direction forms the angle $\alpha$ with the optic axis. This refractive index is given by the following relation: ${ }^{26}$

$$
\frac{1}{n^{2}(\alpha)}=\frac{\cos ^{2} \alpha}{n_{o}^{2}}+\frac{\sin ^{2} \alpha}{n_{e}^{2}}
$$

where $n_{e}$ is the extraordinary refractive index of the uniaxial crystal.

For small values of $\alpha$, the ordinary and the extraordinary waves practically propagate along the same direction, so that they travel almost the same distance $d_{e} \simeq d_{0}=d=\sqrt{l^{2}+r^{2}}$. At the exit plane the ordinary and the extraordinary waves can be decomposed in their $x$ and $y$ components, and the field can be expressed as ${ }^{4}$

$$
\mathbf{E}(r, \theta)=\left(\begin{array}{c}
E_{x}(r, \theta) \\
E_{y}(r, \theta)
\end{array}\right) \simeq E_{0}(r, \theta) \exp \left(\mathrm{i} k \frac{n(\alpha)+n_{o}}{2} d\right)\left(\begin{array}{c}
\cos ^{2} \theta \exp (\mathrm{i} \delta(r) / 2)+\sin ^{2} \theta \exp (-\mathrm{i} \delta(r) / 2) \\
\cos \theta \sin \theta[\exp (\mathrm{i} \delta(r) / 2)-\exp (-\mathrm{i} \delta(r) / 2)]
\end{array}\right),
$$

where the phase $\delta(r)$ is ${ }^{26}$

$$
\delta(r)=k n(\alpha) d_{e}-k n_{o} d_{o} \simeq k\left[n(\alpha)-n_{o}\right] d \simeq k\left(n_{e}-n_{o}\right) d \sin ^{2} \alpha=k\left(n_{e}-n_{o}\right) \frac{r^{2}}{d} .
$$

Results in Eqs. (3) and (4) have been obtained for the case of a point-like source in a quite easy way, through a geometrical-optics approach. A more complex derivation, based on the plane-wave decomposition of a field, was presented in Refs. ${ }^{27,28}$ for the propagation of Gaussian beams along the optic axis of a uniaxial crystal. It can be shown that the latter approach leads to comparable numerical results when a fundamental Gaussian beam, having waist size small enough, is considered.

The polarization state of the field at a typical point across the exit plane can be described by means of the Stokes parameters, ${ }^{26}$ derived from the electric field as

$$
\mathbf{S}(r, \theta)=\left(\begin{array}{c}
S_{0}(r, \theta) \\
S_{1}(r, \theta) \\
S_{2}(r, \theta) \\
S_{3}(r, \theta)
\end{array}\right)=\left(\begin{array}{c}
\left|E_{x}(r, \theta)\right|^{2}+\left|E_{y}(r, \theta)\right|^{2} \\
\left|E_{x}(r, \theta)\right|^{2}-\left|E_{y}(r, \theta)\right|^{2} \\
2 \operatorname{Re}\left\{E_{x}(r, \theta) E_{y}^{*}(r, \theta)\right\} \\
-2 \operatorname{Im}\left\{E_{x}(r, \theta) E_{y}^{*}(r, \theta)\right\}
\end{array}\right)
$$

where $\operatorname{Re}\{\cdot\}$ and $\operatorname{Im}\{\cdot\}$ denote real and imaginary part, respectively. The first Stokes parameter represents the intensity, the second one is the difference between the contents of polarized light along the $x$ and $y$ directions, the third one is analogous to the second one, but considers the polarized light along the first- and the secondquadrant bisectors, and finally the fourth parameter is the difference between right-handed and left-handed 

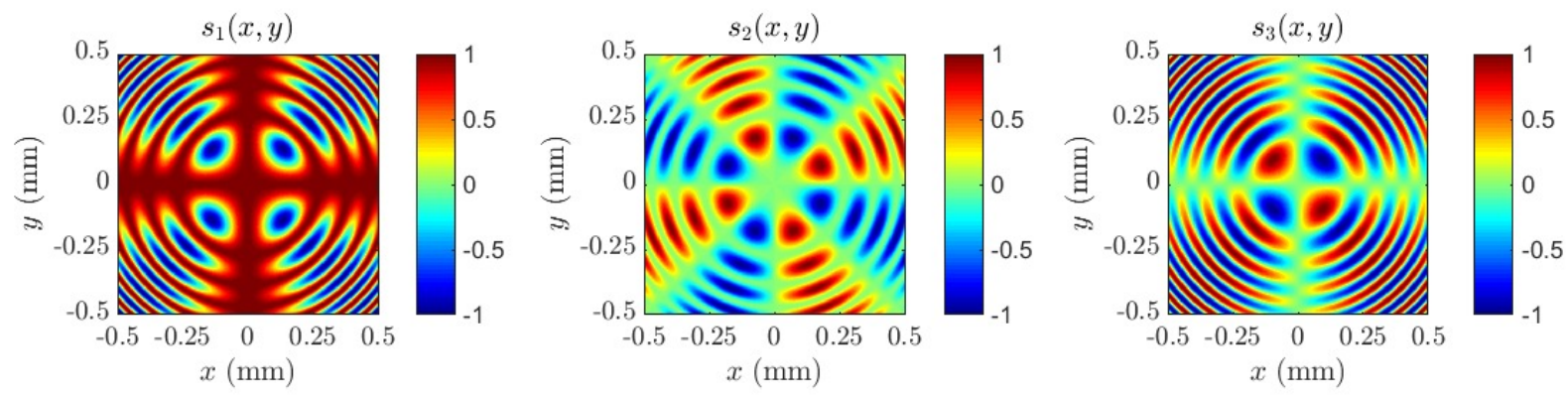

Figure 2. Normalized Stokes parameters at the exit plane of a $20 \mathrm{~mm}$ length calcite crystal and $632.8 \mathrm{~nm}$ wavelength $\left(n_{e}=1.4849, n_{o}=1.6557\right)$.

circularly polarized content of the beam. ${ }^{26}$ For the field given by Eq. (3) the normalized Stokes parameters, defined as $s_{i}(r, \theta)=S_{i}(r, \theta) / S_{0}(r, \theta)(i=1,2,3)$, can be written as

$$
\mathbf{S}(r, \theta)=S_{0}(r, \theta)\left(\begin{array}{c}
1 \\
s_{1}(r, \theta) \\
s_{2}(r, \theta) \\
s_{3}(r, \theta)
\end{array}\right)=S_{0}(r, \theta)\left(\begin{array}{c}
1 \\
\cos ^{2} 2 \theta+\sin ^{2} 2 \theta \cos \delta(r) \\
\sin 2 \theta \cos 2 \theta[1-\cos \delta(r)] \\
-\sin 2 \theta \sin \delta(r)
\end{array}\right) .
$$

showing that the state of polarization varies continuously from point to point across the transverse section of the beam. Furthermore, it can be easily verified that, as is expected, the field is totally polarized because

$$
s_{1}^{2}(r, \theta)+s_{2}^{2}(r, \theta)+s_{3}^{2}(r, \theta)=1 .
$$

Across the exit plane, the phase $\delta(r)$ varies from 0 to $2 \pi$ when the radius $r$ goes from zero to

$$
r_{1}^{2} \simeq \frac{\lambda d}{n_{e}-n_{o}} \simeq \frac{\lambda l}{n_{e}-n_{o}},
$$

with $\lambda$ being the wavelength. For typical values of birefringence for usual uniaxial crystals in the optical region and crystal lengths of the order of some centimeters, the value of $r_{1}$ is of the order of some tenths of a millimeter. Therefore, it is expected that all values of the Stokes parameter present across the exit field will be contained in an area of the order of a squared millimeter.

Figure 2 shows the values of the normalized Stokes parameters $s_{1}, s_{2}$ and $s_{3}$ (in the following we will omit their spatial dependence) for the case of a $20 \mathrm{~mm}$ length calcite crystal. It can be noted that these three parameters vary in the range $[-1,1]$ several times in the represented area. Moreover, the maximum angle $\alpha$ for this geometry is about 2 degrees, so that the paraxial and the other approximations used above turn out to be well justified.

\section{IS THE OUTPUT BEAM A FULL POINCARÉ BEAM?}

As recalled above, a FPB is a non-uniformly totally polarized beam that presents all possible states of polarization across its transverse section. ${ }^{9}$ This means that for any conceivable polarization state across the surface of the Poincaré sphere ${ }^{26}$ it is possible to find at least one point in the cross section of the beam that presents such state.

In order to prove that the beam in Eq. (3) is a FPB we have to show, using Eq. (6), that for any given Stokes vector $\left(1, s_{1}, s_{2}, s_{3}\right)$ representing a possible totally polarized state of polarization, two real angles $\theta$ and $\delta$ can be found such that the field presents that polarization. The relation between $\delta$ and the radial coordinate $r$ is expressed by Eq. (4). Notice that, since the normalized Stokes parameters have to satisfy Eq. (7), there are only two independent parameters, whose values are restricted to the interval $[-1,1]$.

From Eq. (6) we have 


$$
\sin 2 \theta=-\frac{s_{3}}{\sin \delta(r)}
$$

so that $s_{1}$ can be expressed as

$$
s_{1}=1-\frac{s_{3}^{2}}{\sin ^{2} \delta(r)}+\frac{s_{3}^{2} \cos \delta(r)}{\sin ^{2} \delta(r)}=1-\frac{s_{3}^{2}[1-\cos \delta(r)]}{1-\cos ^{2} \delta(r)}=1-\frac{s_{3}^{2}}{1+\cos \delta(r)},
$$

and $\delta(r)$ can be obtained as a function of $s_{1}$ and $s_{3}$ :

$$
\cos \delta(r)=\frac{s_{3}^{2}}{1-s_{1}}-1
$$

On the other hand, from Eq. (6) we have

$$
s_{2}=\frac{1}{2} \sin 4 \theta[1-\cos \delta(r)]
$$

and, substituting from Eq. (11),

$$
\sin 4 \theta=\frac{2 s_{2}}{1-\cos \delta(r)}=\frac{2 s_{2}\left(1-s_{1}\right)}{2-2 s_{1}-s_{3}^{2}} .
$$

Equations (11) and (13), together with Eq. (4) allows to determine the coordinates where the output field presents the required polarization state.

Now we want to show that Eqs. (11) and (13) always give real solutions for the angles $\delta$ and $\theta$, for any possible choice of the Stokes parameters. For the former case, it is sufficient to prove that the following inequality holds:

$$
-1 \leq \frac{s_{3}^{2}}{1-s_{1}}-1 \leq 1
$$

The left-hand-side condition requires

$$
\frac{s_{3}^{2}}{1-s_{1}} \geq 0
$$

and is clearly satisfied, because $s_{1} \leq 1$. The right-hand-side inequality can be written as

$$
s_{3}^{2}-1+s_{1} \leq 1-s 1
$$

or

$$
s_{3}^{2}+2 s_{1}-2 \leq 0 .
$$

From Eq. (7) it turns out that $s_{3}^{2} \leq 1-s_{1}^{2}$ and then

$$
s_{3}^{2}+2 s_{1}-2 \leq-s_{1}^{2}+2 s_{1}-1=-\left(1-s_{1}\right)^{2} \leq 0,
$$

so that Eq. (17) is proved.

To prove that Eq. (13) always gives a real angle $\theta$ we will show that the following inequality is fulfilled for any possible choice of $s_{1}, s_{2}$ and $s_{3}$ :

$$
-1 \leq \frac{2 s_{2}\left(1-s_{1}\right)}{2-2 s_{1}-s_{3}^{2}} \leq 1
$$

It has already been shown that the denominator of the central part of the inequality is positive [see Eq. (17)], so that we can transform it as

$$
-2+2 s_{1}+s_{3}^{2} \leq 2 s_{2}\left(1-s_{1}\right) \leq 2-2 s_{1}-s_{3}^{2} .
$$

The left-hand-side inequality in Eq. (20) can be written as

$$
-2+2 s_{1}+s_{3}^{2}-2 s_{2}\left(1-s_{1}\right) \leq 0
$$


or, taking Eq. (7) into account,

$$
-2+2 s_{1}+1-s_{1}^{2}-s_{2}^{2}-2 s_{2}\left(1-s_{1}\right)=-\left(1-s_{1}+s_{2}\right)^{2} \leq 0,
$$

which is obviously true. In a similar way, the right-hand-side inequality in Eq. (20) can be expressed as

$$
2-2 s_{1}-s_{3}^{2}-2 s_{2}\left(1-s_{1}\right) \geq 0,
$$

or, using again Eq. (7),

$$
2-2 s_{1}-1+s_{1}^{2}+s_{2}^{2}-2 s_{2}\left(1-s_{1}\right)=\left(1-s_{1}-s_{2}\right)^{2} \geq 0,
$$

which is always satisfied.

\section{CONCLUSIONS}

In this work, an easy way to obtain a particular type of non-uniformly polarized beam by using an uniaxial crystal has been proposed. When a point source is placed at the center of the input plane of a uniaxial crystal having the optic axis oriented along the propagation direction of the emitted light, a nonuniformly polarized beam is obtained at the output. Under paraxial approximation, the state of polarization at any point of the cross section of the output beam can be calculated. It has been proved that, for any given state of polarization represented on the surface of the Poincaré sphere, at least one point exists across the cross section of the output beam where the field presents such state of polarization. In other terms, it has been shown that the output beam is a full Poincaré beam.

\section{ACKNOWLEDGMENTS}

Spanish Ministerio de Economía y Competitividad project FIS2016-75147.

\section{REFERENCES}

[1] Freund, I., "Polarization flowers," Optics Communications 199(14), 47 - 63 (2001).

[2] Gori, F., "Polarization basis for vortex beams," J. Opt. Soc. Am. A 18, 1612-1617 (Jul 2001).

[3] Movilla, J., Piquero, G., Martínez-Herrero, R., and Mejías, P., "Parametric characterization of nonuniformly polarized beams," Optics Communications 149(46), 230 - 234 (1998).

[4] Piquero, G. and Vargas-Balbuena, J., "Non-uniformly polarized beams across their transverse profiles: an introductory study for undergraduate optics courses," European Journal of Physics 25(6), 793 (2004).

[5] Niziev, V. G., Chang, R. S., and Nesterov, A. V., "Generation of inhomogeneously polarized laser beams by use of a Sagnac interferometer," Appl. Opt. 45, 8393-8399 (Nov 2006).

[6] Maurer, C., Jesacher, A., Frhapter, S., Bernet, S., and Ritsch-Marte, M., "Tailoring of arbitrary optical vector beams," New Journal of Physics 9(3), 78 (2007).

[7] Martínez-Herrero, R. and Mejías, P. M., "Propagation of light fields with radial or azimuthal polarization distribution at a transverse plane," Opt. Express 16, 9021-9033 (Jun 2008).

[8] Zhan, Q., "Cylindrical vector beams: from mathematical concepts to applications," Adv. Opt. Photon. 1, 1-57 (Jan 2009).

[9] Beckley, A. M., Brown, T. G., and Alonso, M. A., "Full Poincaré beams," Opt. Express 18, 10777-10785 (May 2010).

[10] Galvez, E. J., Khadka, S., Schubert, W. H., and Nomoto, S., "Poincarè-beam patterns produced by nonseparable superpositions of Laguerre-Gauss and polarization modes of light," Appl. Opt. 51, 2925-2934 (May 2012).

[11] Vyas, S., Kozawa, Y., and Sato, S., "Polarization singularities in superposition of vector beams," Opt. Express 21, 8972-8986 (Apr 2013).

[12] Korotkova, O. and Wolf, E., "Changes in the state of polarization of a random electromagnetic beam on propagation," Optics Communications 246(13), 35 - 43 (2005). 
[13] Wang, T. and Pu, J., "Propagation of non-uniformly polarized beams in a turbulent atmosphere," Optics Communications 281(14), 3617 - 3622 (2008).

[14] Ramírez-Sánchez, V., Piquero, G., and Santarsiero, M., "Generation and characterization of spirally polarized fields," Journal of Optics A: Pure and Applied Optics 11(8), 085708 (2009).

[15] Ding, C., Pan, L., and Lü, B., "Changes in the state of polarization of apertured stochastic electromagnetic modified Bessel-Gauss beams in free-space propagation," Applied Physics B 99(1), 307-315 (2010).

[16] Ramírez-Sánchez, V., Piquero, G., and Santarsiero, M., "Synthesis and characterization of partially coherent beams with propagation-invariant transverse polarization pattern," Optics Communications 283(22), 4484 - 4489 (2010). Electromagnetic Coherence and Polarization.

[17] Santarsiero, M., de Sande, J. C. G., Piquero, G., and Gori, F., "Coherencepolarization properties of fields radiated from transversely periodic electromagnetic sources," Journal of Optics 15(5), 055701 (2013).

[18] de Sande, J. C. G., Santarsiero, M., Piquero, G., and Gori, F., "Longitudinal polarization periodicity of unpolarized light passing through a double wedge depolarizer," Opt. Express 20, 27348-27360 (Dec 2012).

[19] de Sande, J. C. G., Piquero, G., and Teijeiro, C., "Polarization changes at Lyot depolarizer output for different types of input beams," J. Opt. Soc. Am. A 29, 278-284 (Mar 2012).

[20] de Sande, J. C. G., Piquero, G., Santarsiero, M., and Gori, F., "Partially coherent electromagnetic beams propagating through double-wedge depolarizers," Journal of Optics 16(3), 035708 (2014).

[21] Zheng, X., Lizana, A., Peinado, A., Ramírez, C., Martínez, J. L., Márquez, A., Moreno, I., and Campos, J., "Compact lcos-slm based polarization pattern beam generator," J. Lightwave Technol. 33, 2047-2055 (May 2015).

[22] Wei, C., Wu, D., Liang, C., Wang, F., and Cai, Y., "Experimental verification of significant reduction of turbulence-induced scintillation in a full Poincaré beam," Opt. Express 23, 24331-24341 (Sep 2015).

[23] Davis, J. A., Moreno, I., Badham, K., Sánchez-López, M. M., and Cottrell, D. M., "Nondiffracting vector beams where the charge and the polarization state vary with propagation distance," Opt. Lett. 41, 2270-2273 (May 2016).

[24] de Sande, J. C. G., Piquero, G., and Santarsiero, M., "Mueller matrix polarimetry by means of azimuthally polarized beams and adapted commercial polarimeter," in [Proceedings of the 5th International Conference on Photonics, Optics and Laser Technology - Volume 1: PHOTOPTICS,], 39-43 (2017).

[25] de Sande, J. C. G., Santarsiero, M., and Piquero, G., "Spirally polarized beams for polarimetry measurements of deterministic and homogeneous samples," Optics and Lasers in Engineering 91, 97 - 105 (2017).

[26] Born, M. and Wolf, E., [Principles of Optics], Cambridge University Press, sixth (corrected) ed. (1980).

[27] Ciattoni, A., Cincotti, G., and Palma, C., "Ordinary and extraordinary beams characterization in uniaxially anisotropic crystals," Optics Communications 195(14), 55 - 61 (2001).

[28] Cincotti, G., Ciattoni, A., and Palma, C., "Hermite-Gauss beams in uniaxially anisotropic crystals," IEEE Journal of Quantum Electronics 37, 1517-1524 (Dec 2001). 